\title{
Influence of long-term equine herpesvirus type 1 (EHV-1) infection on primary murine neurons - the possible effects of the multiple passages of EHV-1 on its neurovirulence
}

\author{
Joanna Cymerys $^{1}$ - A. Slońska ${ }^{1}$ - A. Tucholska ${ }^{1}$ - A. Golke ${ }^{1}$ - A. Chmielewska ${ }^{1}$. \\ M. W. Bańbura ${ }^{1}$
}

Received: 5 April 2016/Accepted: 2 April 2017 /Published online: 13 April 2017

(C) The Author(s) 2017. This article is an open access publication

\begin{abstract}
Equine herpesvirus 1 (EHV-1), like other members of the Alphaherpesvirinae subfamily, is a neurotropic virus causing latent infections in the nervous system of the natural host. In the present study, we have investigated EHV-1 replication (wild-type Jan-E strain and Rac-H laboratory strain) during long-term infection and during the passages of the virus in cultured neurons. The studies were performed on primary murine neurons, which are an excellent in vitro model for studying neurotropism and neurovirulence of EHV-1. Using real-time cell growth analysis, we have demonstrated for the first time that primary murine neurons are able to survive long-term EHV-1 infection. Positive results of real-time PCR test indicated a high level of virus DNA in cultured neurons, and during long-term infection, these neurons were still able to transmit the virus to the other cells. We also compared the neurovirulence of Rac-H and Jan-E EHV-1 strains after multiple passages of these strains in neuron cell culture. The results showed that multiple passages of EHV-1 in neurons lead to the inhibition of viral replication as early as in the third passage. Interestingly, the inhibition of the EHV-1 replication occurred exclusively in neurons, because the equine dermal (ED) cells co-cultivated with neuroculture medium from the third passage showed the presence of large amount of viral DNA. In conclusion, our results showed that certain balance between EHV-1 and neurons has been established during in vitro infection allowing neurons to survive longterm infection.
\end{abstract}

Joanna Cymerys

jcymerys@op.pl

1 Division of Microbiology, Department of Preclinical Sciences, Faculty of Veterinary Medicine, Warsaw University of Life Sciences - SGGW, Ciszewskiego 8, 02-786 Warsaw, Poland

\section{Introduction}

The natural biology of alphaherpesviruses is a primary infection with mild or no symptoms and a highly successful establishment of a long-term relationship with the host. Alphaherpesviruses (e.g. HSV-1, HSV-2, BHV-1, VZV, PRV) establish a life-long latency in peripheral neurons where productive replication is suppressed. Equine herpesvirus 1 (EHV-1), a major causative agent of upper respiratory tract infections and abortion in horses, similarly to other alphaherpesviruses is also neurotropic and causes latent infection in the neurons of natural host (Delhon et al. 2002; Ch'ng and Enquist 2005). Despite the fact that many studies have been devoted to the pathogenesis of various clinical forms of EHV-1 infection, mechanisms of the neuronal damage are not fully understood (Pusterla and Hussey 2014; Slater et al. 1994; Sauerbrei and Wutzler 2002; De Regge et al. 2006; Bańbura et al. 2000). Most of the available information about the latency establishment, maintenance and reactivation of alphaherpesviruses is derived from in vivo studies (Wilson and Mohr 2012; Sauerbrei and Wutzler 2002; Baxi 1995); however, it is difficult to differentiate specific effects of direct virusneuron relationship from indirect consequences mediated by immune or non-neuronal supportive cells. Consequently, presented in vitro model utilizing cultured primary murine neurons provides a simple and effective method to examine the kinetics of EHV-1 replication, to determine the differences in the influence of the virus on the neuronal cell depending on virus strain and its adaptation to the cell culture. Our previous studies had provided answers to a number of key EHV-1-related questions. We found out that the field isolate Jan-E (at low in vitro passage) and reference strain Rac-H (at high in vitro passage) were able to replicate without the need for adaptation in murine neurons in vitro. Moreover, positive real-time PCR (quantitative PCR; qPCR) and nestedPCR (nPCR) results indicated the presence of viral DNA in neurons $24-48 \mathrm{~h}$ post infection (p.i.) with both 
EHV-1 strains; however, some neurons survived infection and showed EHV-1 replication (Cymerys et al. 2010). We also demonstrated that Rac-H and Jan-E EHV-1 strains were able to induce apoptosis in primary murine neurons; however, apoptotic neurons represented only a small percentage of the total cell number. Moreover, the majority of the observed neurons were not only resistant to the EHV-1-induced cell death but also reentered the active proliferation cycle (increase in the number of cells in S and G2M phases of cell cycle) (Cymerys et al. 2012). We also observed an autophagy induction during infection of primary murine neurons with both Jan-E and Rac-H strains of EHV-1. However, chloroquine (autophagy inhibitor) did not affect the level of replication of the virus, quantified with realtime PCR method (Cymerys et al. 2014). These results led us to further studies on kinetics of EHV-1 replication during longterm neuronal infection and to examine the effects of the neuronal passage on EHV-1 neurovirulence.

\section{Materials and methods}

\section{Virus strains}

In the present study, two strains of EHV-1 from the virus collection of the Virology Laboratory of the Department of Preclinical Sciences were used. Jan-E is a field strain isolated from aborted foetus (mare Ezelda, Poland; 12th passage in ED cells) and identified by PCR using gB-specific primers (Borchers and Slater 1993). Rac-H is a reference strain isolated in 1957 from the aborted foetus of mare Heraldia, Poland. This strain has been passaged through a series of cell cultures and nowadays is defined as 'pantropic, non-pathogenic' (Nugent et al. 2006).

\section{Neuronal cell cultures}

Balb/c (H-2 $\left.{ }^{\mathrm{d}}\right)$ mice genetically susceptible to EHV-1 infection (Awan et al. 1990; Gosztonyi et al. 2009) were used to establish primary culture of murine neurons, as described before (Cymerys et al. 2010). Cells were plated onto poly-L-lysine or poly-D-lysine with laminin-coated coverslides at a density of $5 \times 10$ (Baxi et al., 1995) to $10^{4}$ neurons per well $\left(9.6 \mathrm{~cm}^{2}\right)$ and into cell culture flasks $\left(10^{4}\right.$ cells per $25 \mathrm{~cm}^{2}$ flask). Primary murine neurons were cultured in B-27 Neuron Plating Medium (Gibco Life Technologies), consisting of neurobasal medium, B27 supplement, glutamine (200 mmol/L), glutamate $(10 \mathrm{mmol} / \mathrm{L} ; 1.8 \mathrm{mg} / \mathrm{mL})$, antibiotics (penicillin-streptomycin, $1 \%$ ) with $10 \%$ supplement of foetal bovine $(5 \%)$ and equine serum (5\%) (Gibco Life Technologies), and maintained at $37{ }^{\circ} \mathrm{C}$ with $5 \% \mathrm{CO}_{2}$. Four days after plating, the medium was removed and replaced with Neuron Feeding Medium (B-27 Neuron Plating Medium without glutamate).
In this medium, neurons were maintained for the next 6 days prior to further manipulations.

\section{Inoculation of cells}

Primary cultures of murine neurons $\left(10^{5}\right.$ cells per $9.6 \mathrm{~cm}^{2}$ well) were infected with EHV-1 (MOI = 1.0) for $60 \mathrm{~min}$ at $37{ }^{\circ} \mathrm{C}$. After adsorption, the inoculum were removed and replaced with fresh culture medium. Infected cells were incubated for $24,48,72,96,120,144$ and $168 \mathrm{~h}$ and 14 or 21 days at $37{ }^{\circ} \mathrm{C}$ with $5 \% \mathrm{CO}_{2}$.

\section{Real-time cell growth analysis}

Cellular growth, behaviour and morphology of neuronal cells infected with EHV-1 strains were analyzed by JuLITM Br Live Cell Analyzer - system for bright-field analysis (NanoEnTek, Korea 2015). Neuronal cells $\left(10^{5}\right.$ cells per $9.6 \mathrm{~cm}^{2}$ well) were seeded in a 6-well plate and infected with Jan-E or Rac-H EHV-1 strains as described above. Cell-growth images were captured for $160 \mathrm{~h}$ with 7-min interval. Cell confluence analysis, as well as real-time cell growth curve, was generated using JuLI Br PC software. Uninfected cells were used as a negative control. All images were captured at a $\times 40$ magnification.

\section{Passage of virus in primary culture of murine neurons}

Primary cultures of murine neurons $\left(10^{5}\right.$ cells per $25 \mathrm{~cm}^{2}$ flask $)$ were infected with EHV-1 (MOI = 1.0) (Rac-H or Jan-E strain) for $60 \mathrm{~min}$ at $37{ }^{\circ} \mathrm{C}$. After adsorption, the inoculum was removed and replaced with fresh culture medium. Subsequently, cells were incubated for $48 \mathrm{~h}$ at $37{ }^{\circ} \mathrm{C}$ with $5 \% \mathrm{CO}_{2}$ and then infected neurons were frozen in $-20{ }^{\circ} \mathrm{C}$ (passage 0 ). The cultures were then thawed and the lysates used for infection of fresh 7-day-old cultures of murine neurons (passage I). The entire cell lysate from passage 0 was used as inoculum. Following $60 \mathrm{~min}$ of adsorption at $37^{\circ} \mathrm{C}$, the inoculum was removed, replaced with fresh culture medium, and the cultures were maintained at $37^{\circ} \mathrm{C}$ in a humidified atmosphere with $5 \% \mathrm{CO}_{2}$ for $48 \mathrm{~h}$. This process was repeated for further nine passages. Neuronal cultures infected directly with the EHV-1 (Jan-E or Rac-H strain), propagated in ED cell cultures (equine dermal cell line; American Tissue Culture Collection, ATCC No. CCL57), served as positive controls. Uninfected neurons served as negative controls.

\section{Real-time PCR}

The quantity of the EHV-1 DNA in all samples was estimated using quantitative real-time PCR (qPCR) with fluorescent TaqMan probes, complementary to a sequence within the amplified products. Samples were collected at 1, 2, 6, 7, 11, 14 
and 21 days p.i. and during multiple passages of EHV-1 strains in neurons. Viral DNA was isolated separately from cells and cell culture medium, using High Pure Viral Nucleic Acid Kit ${ }^{\circledR}$ (Roche Diagnostics), as instructed by the manufacturer. Real-time PCR tests were run on LightCycler 2.0 instrument (Roche Diagnostics, Germany), using a modified inhouse quantitative method, described below (Dzieciątkowski et al. 2009). Highly conservative region encoding glycoprotein $\mathrm{B}(\mathrm{gB})$ gene has been chosen, and set of primers was developed, as well as the probe, labelled with fluorophore reporter JOE on $5^{\prime}$ end and with BHQ-2 quencher on its $3^{\prime}$ end (Oligo, Poland). Investigations were performed using reaction mixture TaqMan Master Kit ${ }^{\circledR}$ (Roche Diagnostics, Germany). Each amplification reaction embraced, except tested samples, also positive calibrators in range 100-1,000,000 copies per $\mathrm{mL}$ and negative control of DNA extraction and amplification process. In order to assess the sensitivity of assay, plasmid construct was developed by cloning the fragment of EHV-1 gB gene (328 bp) into SmaI digested pBluescript II SK(-) (Epoch LifeScience, USA). The concentration of obtained amplicon DNA was determined spectrophotometrically by absorbance of UV light at 260 and $280 \mathrm{~nm}$. The limit of detection (LOD) of modified qPCR assay was determined by analysis of serial decimal dilutions of amplicon DNA in range $10^{1}-10^{6}$ copies. Each dilution was prepared and analyzed in six independent replications. Probit analysis was used to calculate the LOD concentration (Burns and Valdiva 2008). Fluorescence levels were measured at $560-\mathrm{nm}$ wavelength, and a threshold cycle $(C t)$ value for each sample was calculated. $C t$ values of EHV-1 calibrators were the basis for standard curves, and the copy numbers were calculated automatically by a software package for data analysis. LOD of used qPCR assay, established on level 227 copies per $\mathrm{mL}$, was adopted for this study as a cut-off value.

\section{Statistical analysis}

The results were statistically evaluated by one-way analysis of variation (ANOVA) using the Student-Newman-Keuls multiple comparisons test and the Turkey-Kramer multiple comparisons test by GraphPad PrismTM version 4.03 software (GraphPad Software Inc., San Diego, CA, USA). All experiments were repeated at least three times. Statistical differences were interpreted as significant at $P \leq 0.05(*)$, highly significant at $P \leq 0.01(* *)$ and not significant at $P \geq 0.05$.

\section{Results}

\section{Morphology of neurons infected with EHV-1}

Real-time cell growth analysis was conducted using live cell movie analyser JuLI Br. During the first $24 \mathrm{~h}$ (Fig. 1a), large numbers of adherent neuronal cell bodies were observed. Development of neuronal projections and axon branching were noticed at $72 \mathrm{~h}$ (Fig. 1c). During imaging 96-160 h (Fig. 1d-f), neurons continued to extend and formed a dense, intact fibre network, radiating from the neuronal cell bodies through the dendrites and axon. This observation was confirmed by detecting cell confluence and creating growth curve using image base analysis (Fig. 1g). Similar analyses were conducted for positive controls-equine dermal (ED) cells infected with Jan-E EHV-1 (Fig. 2). During Jan-E EHV-1 infection, a cytopathic effect (CPE) was visible as early as $24 \mathrm{~h}$ p.i. and manifested by drastically changed morphology and degeneration of cells, which led to focal degeneration (Fig. 2b-f). Between 24 and 38 h p.i., a significant decrease from 98.35 to $29.02 \%$ in the level of confluence was noticed (Fig. 2g).

In neurons infected with Jan-E strain, destruction of cells and focal degeneration was also observed, however not so evident as in ED cells (Fig. 3). At the beginning, confluence of the culture decreased from $79 \%$ ( 0 h p.i.) to $51.73 \%$ ( $33 \mathrm{~h}$ p.i.) and then gradually began to increase up to $68.64 \%$ in $140 \mathrm{~h}$ p.i. (Fig. 3j). Changes in the morphology of infected neurons were apparent, but some of them remained unchanged and retained their projections (Fig. $3 \mathrm{c}-\mathrm{h}$ ). Moreover, at the empty surface inside the plaques, new single cells were identified (Fig. 3i, k; arrow).

In neurons infected with Rac-H strain, CPE was not as clearly visible as in neurons infected with Jan-E EHV-1 strain. Even though there was no change in the confluence of the culture (Fig. $4 \mathrm{j}$ ), the differences in cell morphology were visible. CPE was manifested by rounding of the infected cell and fusion with adjacent cells to form syncytia (Fig. 4a-i). However, infected cells did not undergo lysis even at $160 \mathrm{~h}$ p.i.

In order to determine how long neurons were able to survive, they were left infected with Jan-E or Rac-H EHV-1 for 8 weeks. After that time, neurons were still viable and CPE was not significantly different from this observed in $160 \mathrm{~h}$ p.i. In cultures infected with Jan-E strain, cells were rounded and devoid of neuronal projections (Fig. 5b). On the other hand, in neuronal culture infected with Rac-H strain, focal degeneration (Fig. 5c) as well as neurons within the plaque were observed (Fig. 5d).

\section{EHV-1 replication during long-term infection of primary murine neurons}

Real-time PCR was applied to detect viral DNA in neuronal cells and in neuroculture medium during long-term infection with Jan-E and Rac-H EHV-1 strains (1, 2, 6, 7, 11, 14 and 21 days p.i.). During infection of neurons with Rac-H reference strain, a significant decrease of copy number of viral DNA was observed from 24 h p.i. $\left(2.94 \pm 0.59 \times 10^{4}\right)$ to 7 days p.i. $\left(1.28 \pm 0.89 \times 10^{4}\right)($ Fig. $6 \mathrm{a})$. In comparison to the uninfected 
Fig. 1 Morphology of uninfected primary murine neurons. Realtime cell growth analysis was performed using live image move analyzer JuLI ${ }^{\mathrm{TM}} \mathrm{Br}$. Cultures were observed from initial seeding for $160 \mathrm{~h}(\mathbf{a}-\mathbf{f})$. All images were recorded every $7 \mathrm{~min}$ and analyzed monolayer confluence (g). Objective magnification $\times 40$
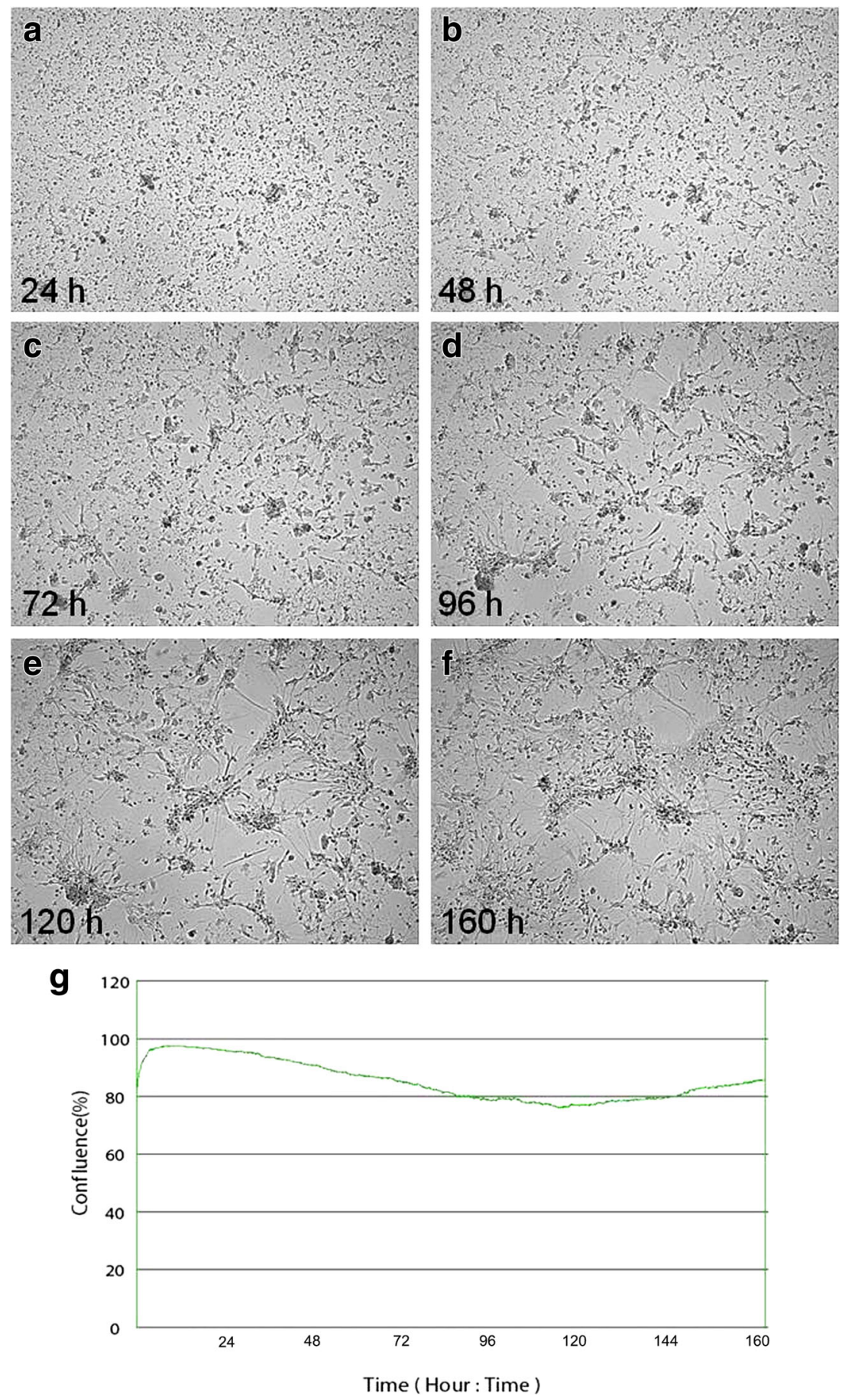

control, the highest copy number of viral DNA was observed at 48 h p.i. $\left(1.0 \pm 0.27 \times 10^{5} ; P \leq 0.01\right)$. Subsequently, 11 and 14 days p.i., a decrease in the copy number of viral DNA was observed in comparison to the first $24 \mathrm{~h}$ of infection (from $1.70 \pm 0.68 \times 10^{3}$ to $1.69 \pm 0.32 \times 10^{2}$ ). Significantly, after 21 days p.i., the copy number of viral DNA increased to the level observed at $24 \mathrm{~h}$ p.i. $\left(3.15 \pm 1.57 \times 10^{4}\right)$. In order to determine the dynamics of replication of Rac-H strain in primary murine neurons, the copy number of viral DNA released to cell culture medium was examined starting from $24 \mathrm{~h}$ p.i. The results showed that the levels of viral DNA released to cell culture medium during long-term infection of neurons with Rac-H strain remained comparatively constant starting from $24 \mathrm{~h}$ p.i. (from $1.41 \pm 1.09 \times 10^{4}$ in $24 \mathrm{~h}$ p.i. to $2.24 \pm 0.92 \times 10^{4}$ in 21 days p.i.; $P \leq 0.05$ ). However, at 11 and 14 days p.i., a decrease of the viral DNA level in the culture medium was observed (from $5.82 \pm 1.08 \times 10^{3}$ to $\left.5.42 \pm 0.81 \times 10^{2}\right)$. 
Fig. 2 Morphological changes of ED cells infected with Jan-E EHV-1 strain. Cells were observed for $48 \mathrm{~h}$ through live image move analyzer JuLI ${ }^{\mathrm{TM}} \mathrm{Br}$ (a-f). CPE was manifested by cell destruction and focal degeneration, confirmed by growth curve $(\mathbf{g})$. Objective magnification $\times 40$
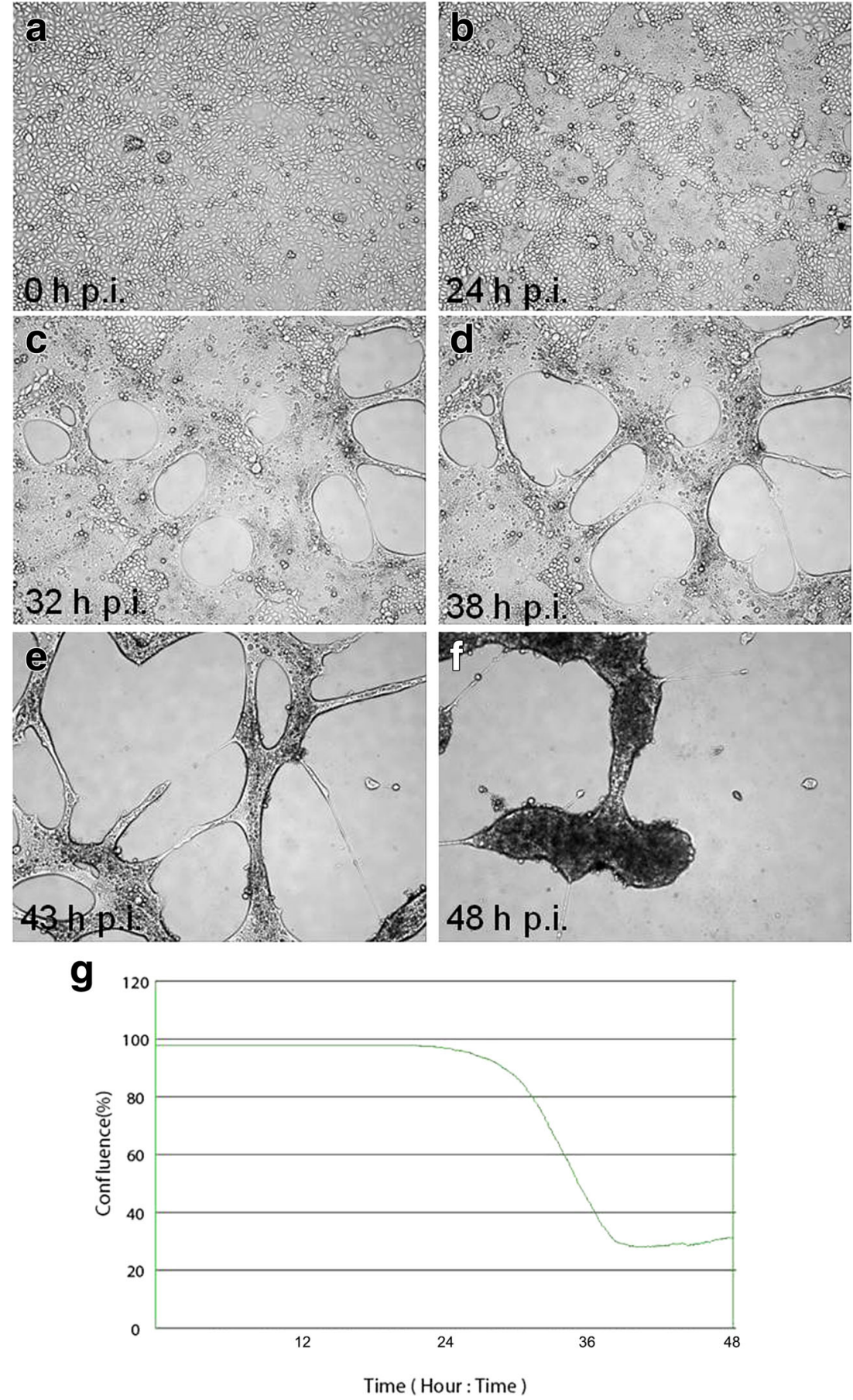

After infection with Jan-E strain of EHV-1, the highest copy number of viral DNA was observed $24 \mathrm{~h}$ p.i. During the course of infection, we observed a decrease in viral DNA copy number reaching the lowest levels at 11 and 14 days p.i. (from $3.31 \pm 1.27 \times 10^{3}$ to $2.86 \pm 0.51 \times 10^{2}$ ). Afterwards, at 21 days p.i., the copy number of viral DNA increased $\left(1.53 \pm 0.89 \times 10^{4}\right)$ and was detected at a level comparable to the level detected at $24 \mathrm{~h}$ p.i. The copy number of viral DNA in culture medium remained at a constant level throughout the course of infection, ranging from $24 \mathrm{~h}$ p.i. to
21 days p.i. (from $1.56 \pm 0.93 \times 10^{4}$ to $1.1 \pm 1.39 \times 10^{4}$; $P \leq 0.05)$.

\section{The effects of EHV-1 passage in primary murine neurons}

The results of passaging Rac-H and Jan-E strains of EHV-1 in primary murine neurons showed that only in the zero passage (0), which simultaneously constituted a positive control, a statistically significant increase in the copy number of viral DNA (Rac-H $2.71 \pm 1.02 \times 10^{4}$; Jan-E 

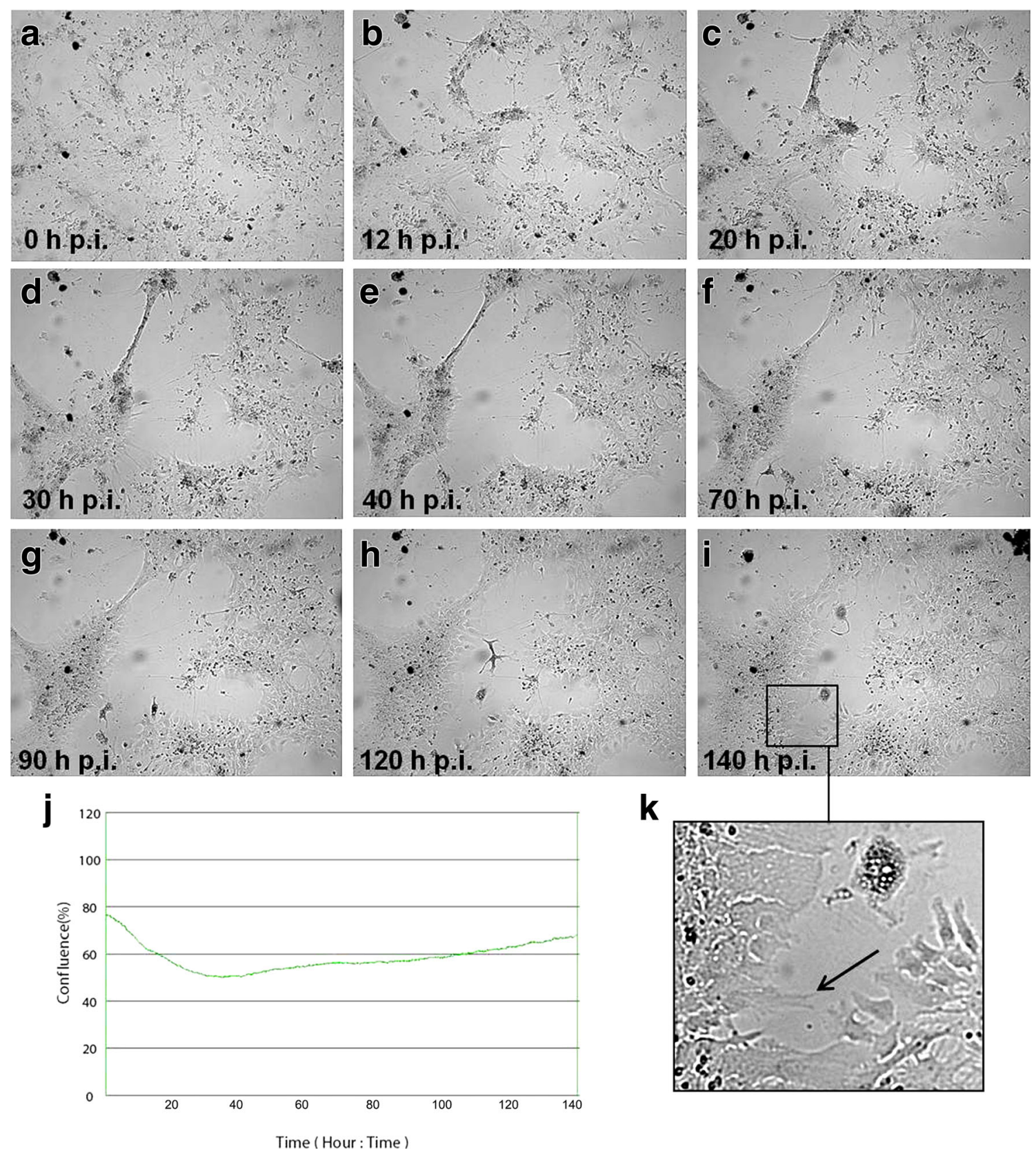

k

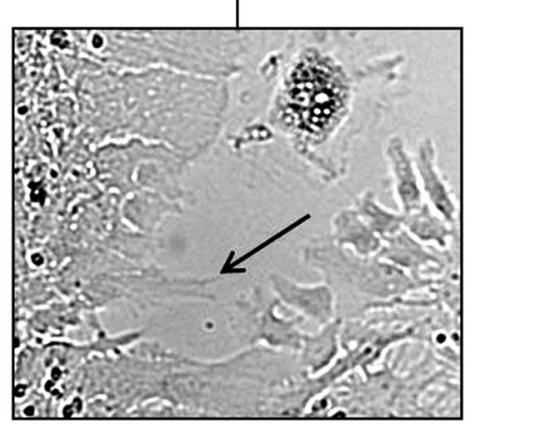

Time ( Hour : Time)

Fig. 3 Morphological changes of primary murine neurons infected with Jan-E EHV-1 strain. Neurons were observed for $140 \mathrm{~h}$ through live image move analyzer $\mathrm{JuLI}^{\mathrm{TM}} \mathrm{Br}(\mathbf{a}-\mathbf{i})$. CPE was manifested by cell destruction and focal degeneration, confirmed by growth curve (j), but not so evident

as in ED cells infected with Jan-E EHV-1. At the free surface inside the plaques, single cells were observed (k, arrow). Objective magnification $\times 40$

$\left.3.20 \pm 1.12 \times 10^{4} ; P \leq 0.01\right)$ was observed in comparison to uninfected control (Fig. 7). In the first passage (I), EHV-1 replication was also observed; however, the copy number of viral DNA was significantly lower than in the zero passage $\left(\right.$ Rac-H $3.20 \pm 0.14 \times 10^{2}$; Jan-E $1.12 \pm 0.42 \times 10^{3}$; $P \leq 0.05$ ). In the second passage (II), viral DNA was still detected in neurons; however, the results were not statistically significant in comparison to the uninfected control. Interestingly, beginning from the fourth passage (IV) (Rac$\mathrm{H}$ and Jan-E) to the tenth passage (X), viral DNA was not present in neurons at a detectable level. For that reason, we decided to infect equine dermal (ED) cells with the neuroculture medium from the third passage (III) of EHV1 in neurons (Rac-H $230 \pm 0.49$ copies per mL; Jan-E $241 \pm 0.51$ copies per $\mathrm{mL}$ ). After $24 \mathrm{~h}$ of incubation, a statistically significant increase in the copy number of viral DNA for both Rac-H and Jan-E strains was observed in ED cells in comparison to neurons from the third passage (RacH $4.17 \pm 1.02 \times 10^{4}$; Jan-E $\left.2.69 \pm 1.51 \times 10^{5} ; P \leq 0.01\right)$ (Fig. 8). 

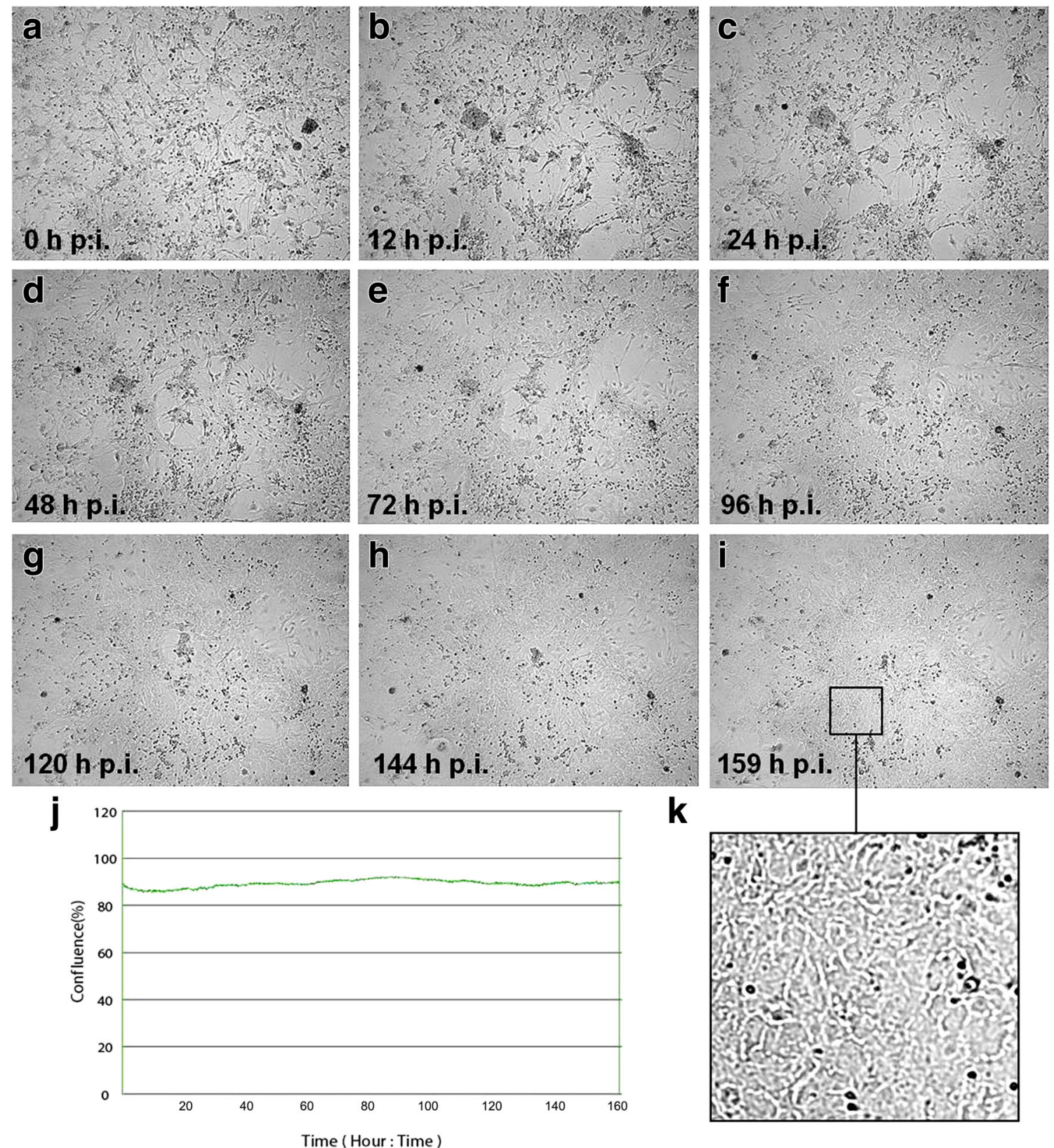

Fig. 4 Morphological changes of primary murine neurons infected with Rac-H EHV-1 strain. Neurons were observed for $160 \mathrm{~h}$ through live image move analyzer JuLI ${ }^{\mathrm{TM}} \mathrm{Br}(\mathbf{a}-\mathbf{i})$. CPE was not as obvious as in neurons infected with Jan-E EHV-1 strain. There was no change in the

level of confluence (j); however, rounding of the infected cell and fusion with adjacent cells to form syncytia was visible (k). Objective magnification $\times 40$

\section{Discussion}

The problem with selection of proper experimental model should be strongly emphasized in the context of the research on EHV-1 infections. As we mentioned in our previous paper, the use of natural host is very limited due to the difficulties associated with finding "immunologically naive" test animals that have not been in contact with the virus (Cymerys et al. 2010). Pusterla and Hussey (2014) indicated that more than $80 \%$ of horses are estimated to be latently infected with EHV-
1. Moreover, other equine herpesviruses, such as EHV-2 or EHV-5, may affect the results of the experiment (Borchers et al. 1999; Ruszczyk et al. 2001; Osińska et al. 2012). For that reason, BALB/c mouse strain in which EHV-1 infection is similar to that in the natural host constitutes a suitable research model (Bańbura et al. 2000; Awan et al. 1990; Gosztonyi et al. 2009; Walker et al. 1999). Results presented by the other authors provide a strong evidence that EHV-1 causes latent infection in mice with the presence of viral DNA not only in mononuclear cells but also in neurons of the olfactory bulbs 
Fig. 5 CPE in primary murine neurons infected with EHV-1 strains (8 weeks p.i.). Microscope image of uninfected neurons (a) and cells infected with Jan-E (b) and Rac-H (c) EHV-1. After that time, single neurons within the plaque were still observed (d). Magnification $\times 200$
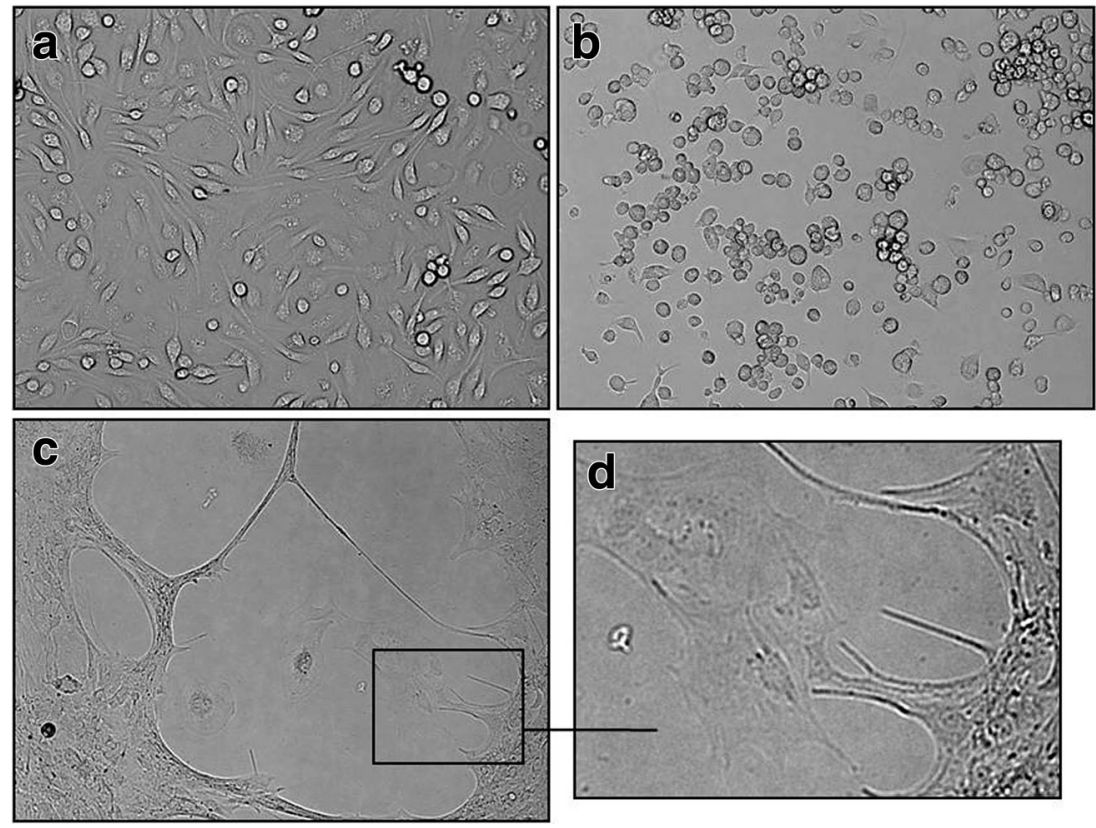

a

Fig. 6 Real-time PCR analysis of viral DNA level during long-term neurons infection. Comparison of viral DNA level (viral gB copy number per $\mathrm{mL}$ ) in primary murine neurons and cell culture medium during 21 days post infection with Rac-H (a) and JanE (b) strains of EHV-1. Statistical differences were interpreted as significant at $P \leq 0.05(*)$, highly significant at $P \leq 0.01(* *)$ and not significant at $P \geq 0.05$

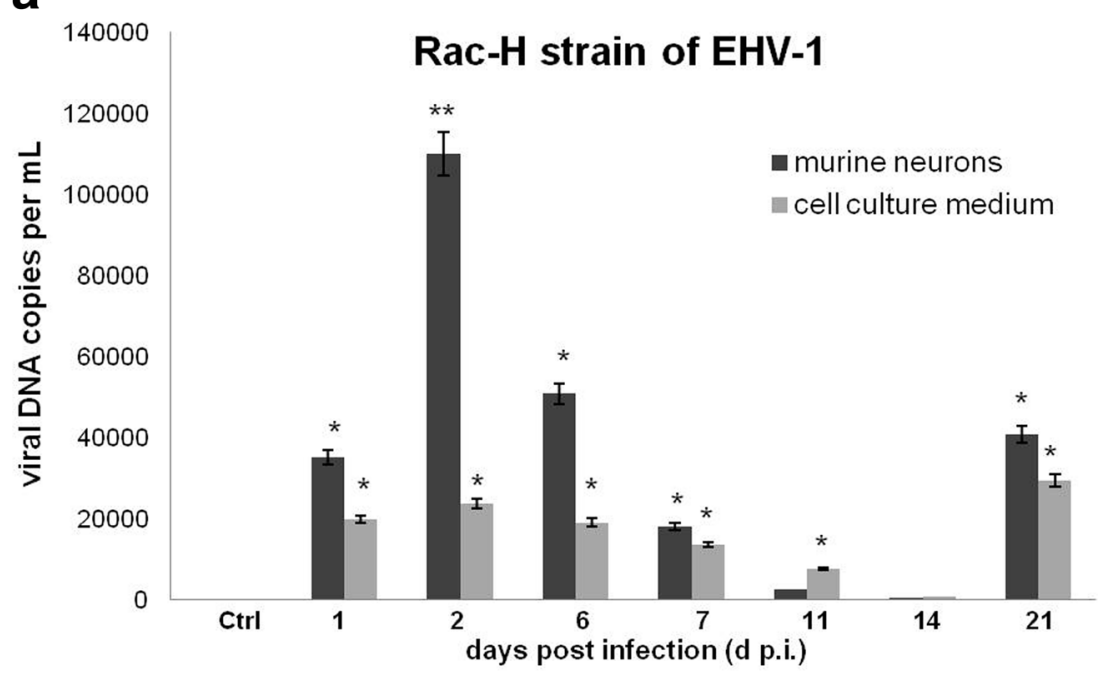

b

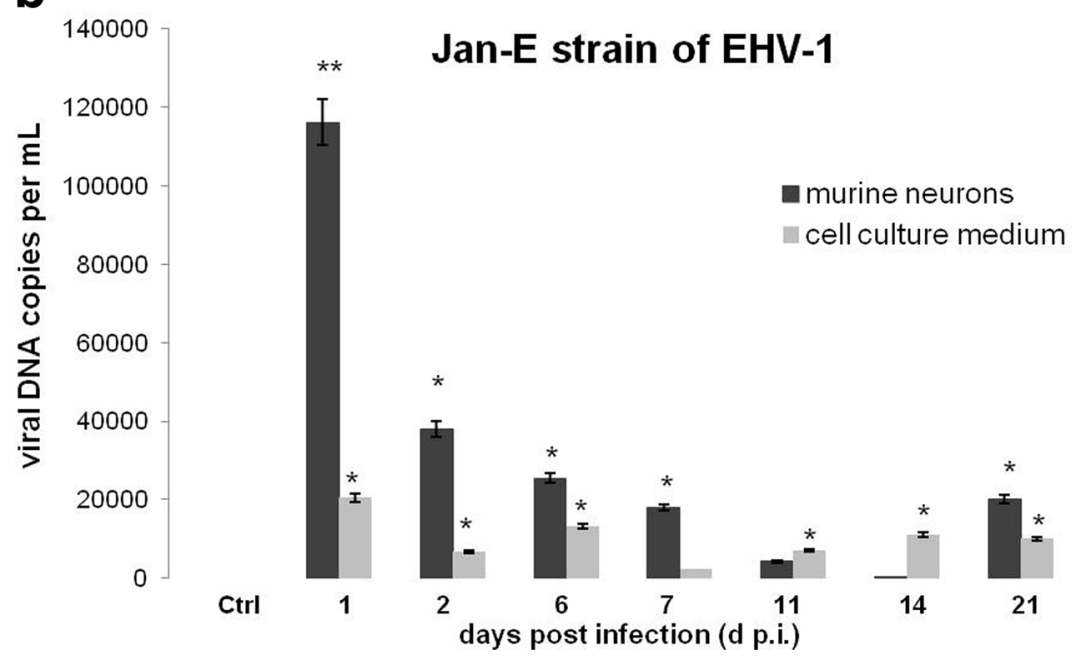


Fig. 7 Real-time PCR analysis of viral DNA level during multiple passages of EHV-1 strains. The neurons-to-neurons EHV-1 (Rac$\mathrm{H}$ and Jan-E strain) passage was repeated ten times. Significant level of viral DNA (viral gB copy number per $\mathrm{mL}$ ) was observed only in the zero (0) and the first (I) passages. Statistical differences were interpreted as significant at $P \leq 0.05(*)$, highly significant at $P \leq 0.01$ (**) and not significant at $P \geq 0.05$

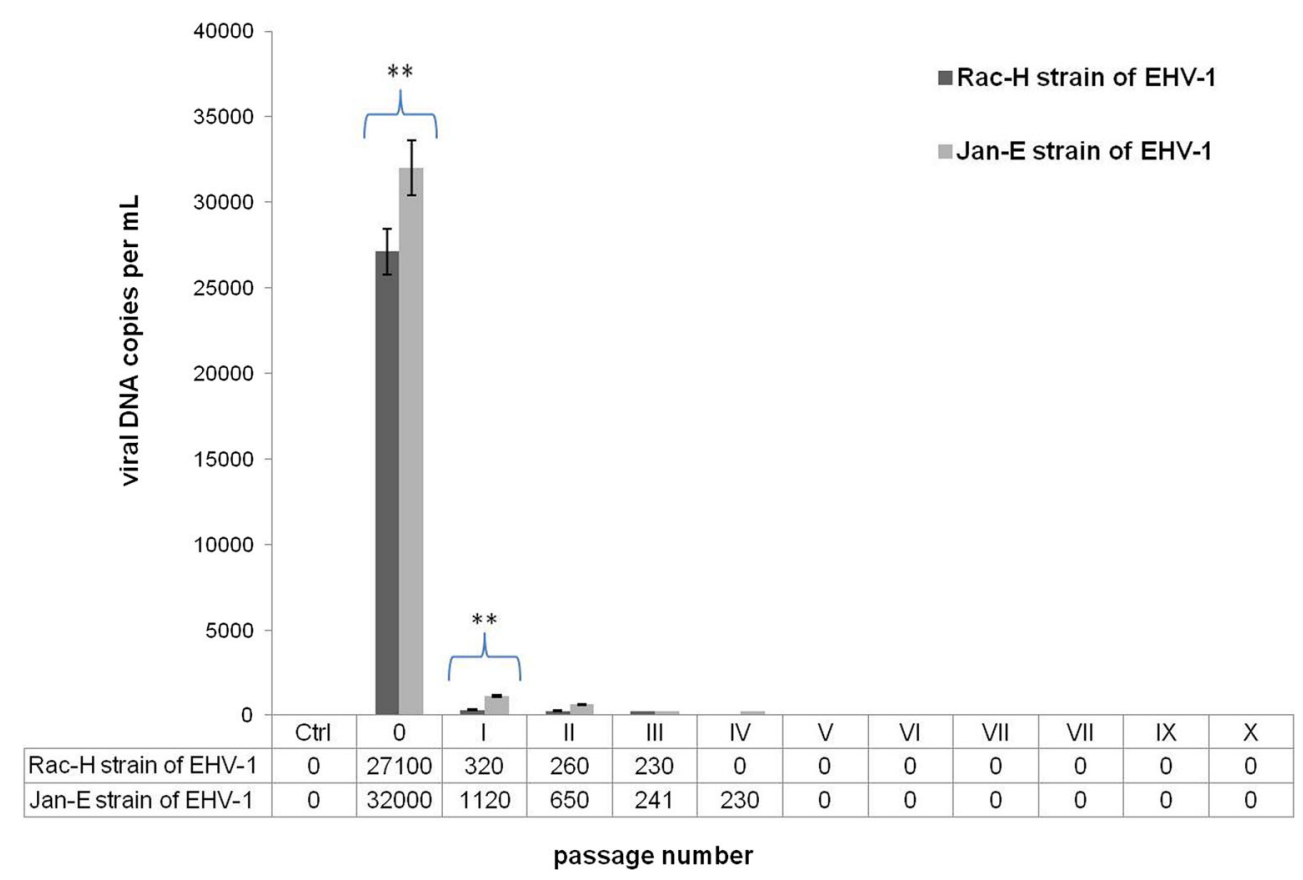

and trigeminal ganglion (Bańbura et al. 2000; Baxi et al. 1995; Marshall and Field 1997). Therefore, it is crucial to clarify what kind of processes take place during the neurological episode, especially during long-term infection with EHV-1. For that reason, presented here in vitro model utilizing cultured primary murine neurons provides a simple and effective method to examine the kinetics of EHV-1 replication and to determine differences between EHV-1 strains and allows to investigate the specific virus-neuron relationship, including long-term EHV-1 infection. Using real-time cell growth analysis, during the early stages of infection, we observed the occurrence of CPE in primary murine neuron culture after infection with both Jan-E and Rac-H strains of EHV-1 (Figs. 3 and 4). These changes were best characterized by abnormal cell morphology, e.g., shrunken, rounded and distorted appearance. CPE is typical for cells which undergo lysis due to viral infection and that indicate productive infection. Jan-E (field strain) induced CPE within $48 \mathrm{~h}$ after infection of murine neuronal cells. On the other hand, infection with $\mathrm{Rac}-\mathrm{H}$ (reference strain) led to similar result within $96 \mathrm{~h}$. These
Fig. 8 ED cells co-cultivated with neuroculture medium from the third passage. Real-time PCR analysis of viral DNA level (viral $\mathrm{gB}$ copy number per $\mathrm{mL}$ ) in $\mathrm{ED}$ cells infected with the neuroculture medium from the third passage (III) of EHV-1 in neurons. Statistically significant $(P \leq 0.01, * *)$ increase in the copy number of viral DNA for both Rac-H and Jan-E strain was observed in ED cells after $24 \mathrm{~h}$ p.i.

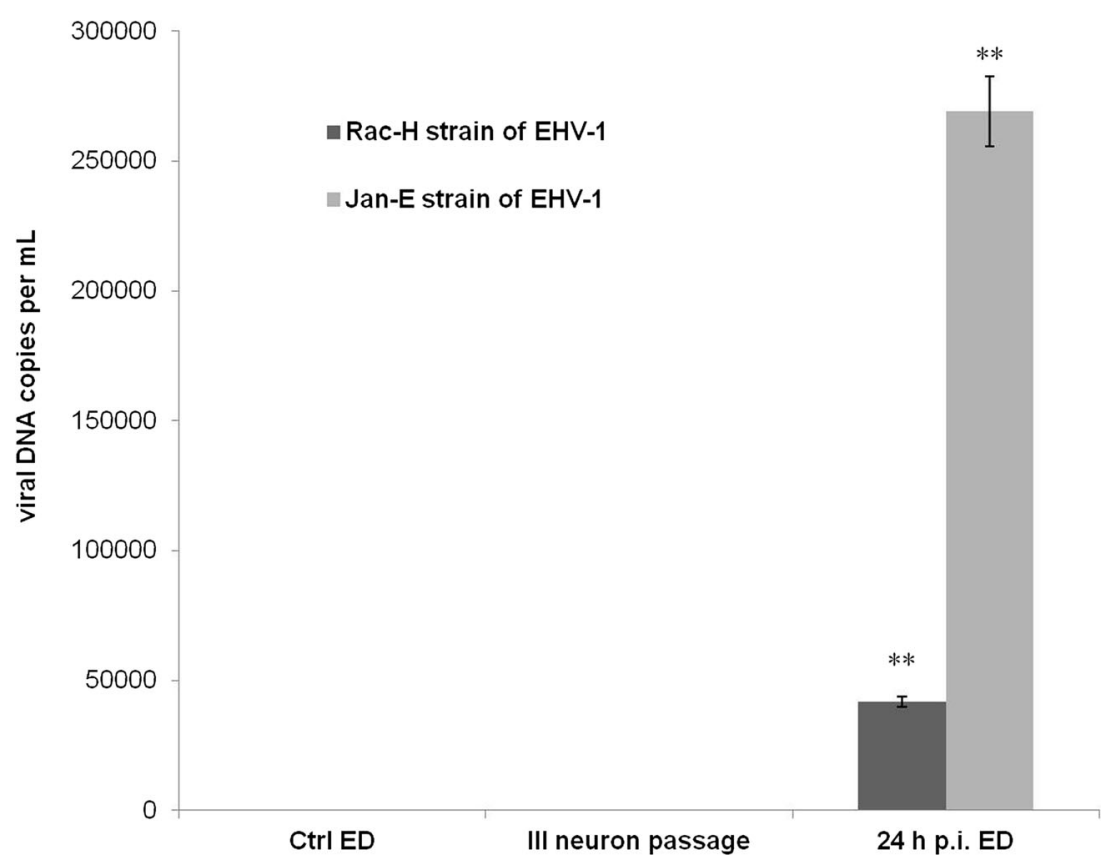


findings were confirmed by real-time PCR results, which showed the largest copy number of viral DNA in primary murine neurons at 24 and 48 h p.i. for Jan-E strain and 48 and $96 \mathrm{~h}$ p.i. in the case of Rac-H strain (Fig. 6). We previously confirmed the presence of EHV-1 antigen within neurons, using immunofluorescence staining, and also proved that EHV-1 can be transported from neuron to neuron (Cymerys et al. 2010; Słońska et al. 2014). After infection of primary murine neurons with Jan-E strain, rearrangements of the actin distribution and formation of long, thin or short, wide actin-containing cell projections were observed. These projections stretched from cell to cell contributing to direct spread of virus particles to adjacent cells without release from the cell. In the case of Rac-H infection, the formation of long projections was not observed (Słońska et al. 2014). However, it should be noted that the CPE caused by Rac-H in primary murine neurons has a different form than that caused by JanE strain. Rac-H does not cause the formation of large free spaces in cell culture, the cells remain closely arranged, which also allows for the transport of the virus from cell to cell (Fig. 4). Interestingly, the differences in actin cytoskeleton rearrangement and the associated direct transport of virions between cells were also partially reflected in the results of real-time PCR. Both for Jan-E and Rac-H strains, the copy number of viral DNA was higher in cells than in the cell culture medium, but apparently for the Rac-H strain, more progeny virions were released from cells than in the case of Jan-E strain (Fig. 6).

In our previous studies, we demonstrated that, despite CPE occurrence, the infection of murine neurons with either of EHV-1 strains (Rac-H or Jan-E) induced changes associated with apoptotic cell death such as DNA fragmentation, chromatin condensation, membrane blebbing and cell shrinkage (Cymerys et al. 2012). However, despite evident changes in the morphology of Jan-E infected neurons, some of them remained unchanged and retained their neuronal projections (Fig. 3c-h). Moreover, at the empty surface inside the plaques, single neurons were identified (Fig. 3i, k; arrow). In neurons infected with Rac-H strain, CPE was manifested by rounding of the infected cell and fusion with adjacent cells to form syncytia (Fig. 4a-i), but the cells did not undergo lysis even at $166 \mathrm{~h}$ p.i. Some neurons in EHV-1 infected cultures changed their shape and degenerated, while some remained morphologically unchanged. The former were presumed to undergo lytic infection with the virus. The latter may have been resistant to EHV-1 infection, or EHV-1 infection was either abortive or latent in such neurons. Further investigation using in situ labelling of viral proteins (IHC or IF) or nucleic acids (ISH) would be necessary to distinguish between these possibilities. These findings confirmed our previous studies, in which we demonstrated that $60-80 \%$ of neurons did not undergo lysis despite the infection (Cymerys et al.
2012) and we suggested that EHV-1 is responsible for suppression of apoptosis in neurons. The question remains, what mechanisms allow neurons to survive during longterm EHV-1 infection. According to our previous research, which revealed the inhibition of apoptosis in EHV-1infected neurons, we concluded that the control of apoptosis may be the key mechanism regulating the balance between productive and latent infection at the site of virus persistence; however, this hypothesis requires further investigation (Cymerys et al. 2012).

In the present study, we also compared the neurovirulence of Rac-H and Jan-E EHV-1 strains during multiple passages in neuronal cell culture. The results showed that multiple passages of EHV-1 in neurons lead to the inhibition of viral replication as early as in the third passage (Fig. 7). Interestingly, the inhibition of the EHV-1 replication occurred exclusively in neurons, because the equine dermal cells (ED cell line) infected with neuroculture medium from the third passage showed the presence of large number of viral particles capable of replication (Fig. 8). These results may suggest that even though we have not confirmed viral replication by realtime PCR, virus was apparently present in neurons. This may also suggest that the number of viral particles is insufficient to maintain infection in murine neurons but sufficient for replication in highly susceptible ED cells. It is also noteworthy that during real-time PCR analyses viral DNA (late gene-gB) was detected (from $24 \mathrm{~h}$ p.i. to 21 days p.i.) (Fig. 6).

In conclusion, our results suggest that certain balance between EHV-1 and neurons has been established during in vitro infection allowing neurons to survive long-term infection. It seems to be logical since neurons are the site of latency, and for the virus benefit, they should survive to maintain latent virus. Using real-time cell growth analysis, we have demonstrated for the first time that primary murine neurons are able to survive long-term EHV-1 infection; nevertheless, the question of interactions between the equine alphaherpesviruses and neuronal cells is still pending, and further studies on EHV-1 replication in neurons are necessary.

Acknowledgments This work was supported by grant NN308 028739 from the Polish Ministry of Science and Higher Education. Authors are also grateful to Alab Polska Sp. z.o.o. (Warsaw, Poland) for providing us access to JuLI Br Live Cell Analyzer (NanoEnTek).

Compliance with ethical standards All animals were handled and cared for according to Polish and European Animal Care and Use guidelines and regulations. All procedures involving live animals were approved by a Local Ethics Committee and confirmed to applicable international standards agreement no. 24/2012.

Competing interests The authors declare that they have no competing interests. 
Open Access This article is distributed under the terms of the Creative Commons Attribution 4.0 International License (http:// creativecommons.org/licenses/by/4.0/), which permits unrestricted use, distribution, and reproduction in any medium, provided you give appropriate credit to the original author(s) and the source, provide a link to the Creative Commons license, and indicate if changes were made.

\section{References}

Awan AR, Chong YC, Field HJ (1990) The pathogenesis of equine herpesvirus type 1 in the mouse: a new model for studying host responses to the infection. J Gen Virol 71:1131-1140. doi:10.1099/ 0022-1317-71-5-1131

Bańbura M, Sendecka H, Chmielewska A, Tucholska A (2000) Histopathological changes and expression of latency-related RNA in the central nervous system of mice latently infected with a field isolate of EHV-1. Med Wet 56:804-808

Baxi MK, Efstathiou S, Lawrence G, Whalley JM, Slater JD (1995) The detection of latency-associated transcript of equine herpesvirus 1 in ganglionie neurons. J Gen Virol 76:3113-3118. doi:10.1099/00221317-76-12-3113

Borchers K, Frölich K, Ludwig H (1999) Detection of herpesvirus types 2 and 5 (EHV-1 and EHV-5) in Przewalski's wild horses. Arch Virol 144:771-780

Borchers K, Slater J (1993) A nested PCR for the detection and differentiation of EHV-1 and EHV-4. J Virol Meth 45:331-336

Burns M, Valdiva H (2008) Modelling the limit of detection in real-time quantitative PCR. Eur Food Res Technol 226:1513-1524

Ch'ng TH, Enquist LW (2005) Neuron-to-cell spread of pseudorabies virus in a compartmented neuronal culture system. J Virol 79: 10875-10889. doi:10.1128/JVI.79.17.10875-10889.2005

Cymerys J, Dzieciątkowski T, Słońska A, Bierła J, Tucholska A, Chmielewska A, Golke A, Bańbura MW (2010) Equine herpesvirus type 1 (EHV-1) replication in primary murine neurons culture. Pol J Vet Sci 13:701-708. doi:10.2478/v10181-010-0022-3

Cymerys J, Miszczak D, Słońska A, Golke A, Bańbura MW (2014) Autophagy in cultured murine neurons infected with equid herpesvirus 1. Acta Virol 58:292-295. doi:10.4149/Av-2014-03-292

Cymerys J, Słońska A, Godlewski M, Tucholska A, Chmielewska A, Golke A, Bańbura MW (2012) Apoptotic and necrotic changes in cultured murine neurons infected with equid herpesvirus 1. Acta Virol 56:39-48. doi:10.4149/Av-2012-01-39

De Regge N, Favoreel HW, Geenen K, Nauwynck HJ (2006) A homologous in vitro model to study interactions between alphaherpesviruses and trigeminal ganglion neurons. Vet Microbiol 113:251-255. doi:10.1016/j.vetmic.2005.11.004
Delhon GA, González MJ, Murcia PR (2002) Susceptibility of sensory neurons to apoptosis following infection by bovine herpesvirus type. J Gen Virol 83:2257-2267. doi:10.1099/0022-1317-83-9-2257

Dzieciątkowski T, Przybylski M, Cymerys J, Turowska A, Chmielewska A, Tucholska A, Banbura MW (2009) Equine herpesvirus type 1 quantification in different types of samples by a real-time PCR. Pol J Vet Sci 12:311-315

Gosztonyi G, Borchers K, Ludwig H (2009) Pathogenesis of equine herpesvirus-1 infection in the mouse model. AMPS 117:10-21. doi:10.1111/j.1600-0463.2008.00008

Marshall KR, Field HJ (1997) Demonstration of equine herpesvirus-1 neuronal latency in marine olfactory bulbs using a novel combined in situ PCR and protein synthesis method. Virology 229:297-282. doi:10.1006/viro.1996.8394

NanoEnTek Inc., Korea (2015) http://www.nanoentek.com. Accessed 8 June 2015

Nugent J, Birch-Machin I, Smith KC, Mumford JA, Swann Z, Newton JR, Bowden RJ, Allen GP, Davis-Poynter N (2006) Analysis of equid herpesvirus 1 strain variation reveals a point mutation of the DNA polymerase strongly associated with neuropathogenic versus nonneuropathogenic disease outbreaks. J Virol 180:4047-4060. doi: 10.1128/JVI.80.8.4047-4060.2006

Osińska E, Golke A, Słońska A, Cymerys J, Bańbura MW, Dzieciatkowski T (2012) HybProbes-based real-time PCR assay for rapid detection of equine herpesvirus type 2 DNA. Pol J Vet Sci 15:411-416

Pusterla N, Hussey GS (2014) Equine herpesvirus 1 myeloencephalopathy. Vet Clin North Am Equine Pract 30:489-506. doi:10.1016/j.cveq. 2014.08.006

Ruszczyk A, Chmielewska A, Tucholska A, Bańbura MW (2001) Isolation and identification equine herpesvirus type 2. Med Wet 57:603-606

Sauerbrei A, Wutzler P (2002) Laboratory diagnosis of central nervous system infections caused by herpesvirises. J Clin Virol 25:45-51. doi:10.1016/S1386-6532(02)00033-1

Slater JD, Borchers K, Thackray AM, Field HJ (1994) The trigeminal ganglion is a location for equine herpesvirus- 1 latency and reactivation in the horse. J Gen Virol 75:2007-2016. doi:10.1099/00221317-75-8-2007

Słońska A, Cymerys J, Godlewski MM, Dzieciątkowski T, Tucholska A, Chmielewska A, Golke A, Bańbura MW (2014) Equine herpesvirus type 1 (EHV-1)-induced rearrangements of actin filaments in productively infected primary murine neurons. Arch Virol 159:13411349. doi:10.1007/s00705-013-1949-3

Walker C, Love DN, Whalley JM (1999) Comparison of the pathogenesis of acute equine herpesvirus 1 (EHV-1) infection in the horse and the mouse model: a review. Vet Microbiol 68:3-13. doi:10.1016/S03781135(99)00056-5

Wilson AC, Mohr I (2012) A cultured affair: HSV latency and reactivation in neurons. Trends Microbiol 20:604-611. doi:10.1016/j.tim. 2012.08.005 